ISSN 1112-9867

Available online at

http://www.jfas.info

\title{
STUDY OF THE PROPAGATION OF SHORT PULSE LASER WITH CAVITY \\ USING NUMERICAL SIMULATION SOFTWARE
}

\author{
S. Terniche*, A. Kellou, A. Kermaoui, R. Si Fodil and R. Becheker
}

Laboratoire Electronique Quantique, USTHB, BP 32 El-Alia, Bab Ezzouar Alger

Received: 25 November 2012 / Accepted: 02 December 2012 / Published online: 30 June 2012

\begin{abstract}
The purpose of this representation is to show the potentialities (Computational Time, access to the dynamic and feasibility of systematic studies) of the numerical study of the nonlinear dynamics in laser cavity, assisted by software. We will give as an example, one type of cavity completely fibered composed of several elements and then studying the physical parameters of a pulse propagating into this cavity, determining its characteristics at the output. The results are interesting but we also projects to verify them experimentally by making assemblies similar to this type of cavities.
\end{abstract} Keywords: Optical fiber, short pulse, bandwidth, spectral width.

\section{INTRODUCTION}

La dynamique de la propagation d'une impulsion intense dans une fibre optique est très riche, plusieurs travaux expérimentaux ont été réalisés mettant en évidence les effets non linéaires régissant les propriétés physiques de l'impulsion. La modélisation numérique de ce type de phénomènes étant complexe, vu le nombre important des paramètres intervenants dans l'équation de propagation des impulsions dans des milieux non linéaires. Nous avons utilisé dans cette étude un logiciel commercial (Fiberdesk) qui permet de simuler la propagation d'une impulsion laser courte dans une fibre.

Author Correspondence, e-mail: sterniche@yahoo.fr

ICID: 1020739 
Nous avons pris comme exemple l'étude de la propagation d'une impulsion dans une cavité complètement fibrée constituée de plusieurs éléments. L'objectif était d'optimiser les caractéristiques de l'impulsion à la sortie, notamment le rapport des largeurs spectrales, en variant les paramètres de simulation.

\section{PRESENTATION DU LOGICIEL}

La Figure 1 montre l'interface graphique du logiciel utilisé. Sur la gauche de la fenêtre, nous pouvons introduire les paramètres de propagation dans le guide. La prise en compte de la dispersion chromatique est réalisée soit en introduisant les valeurs des coefficients du développement en série de Taylor de la constante de propagation, soit à partir d'une structure idéale de fibre microstructurée air-silice en indiquant le diamètre des trous d'air $d$ et l'espacement entre les trous. Le profil de l'impulsion de pompe est déterminé dans la fenêtre «create pulse » (Figure 2), dans laquelle nous introduisons les différents paramètres de l'impulsion.

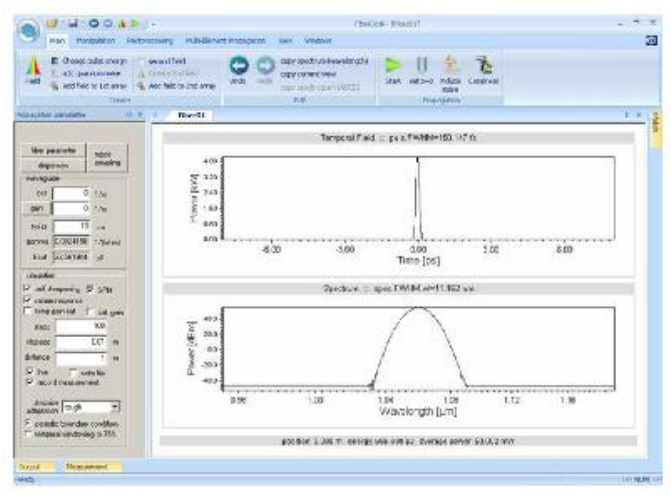

Fig.1. Interface graphique du logiciel comprenant sur la gauche les paramètres de $\mathrm{s}$

propagation, au centre l'évolution $t_{t}$ temporelle et spectrale de l'impulsion

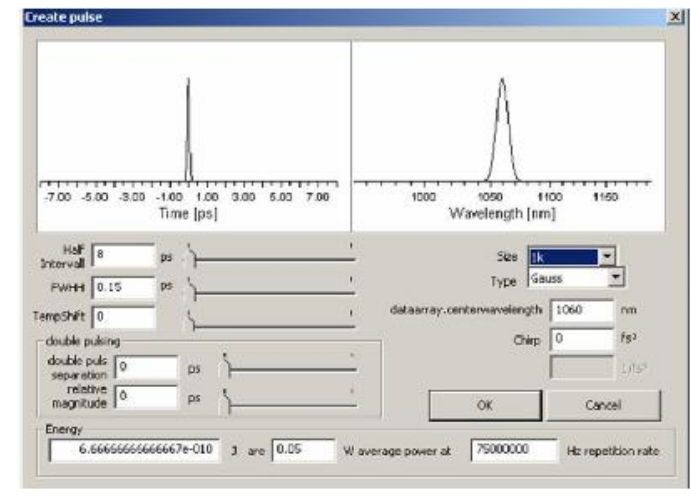

Fig.2. Fenêtre de saisie des caractéristiques de l'impulsion d'entrée

saturable [1]:

$$
\frac{\partial A}{\partial z}=-\frac{\alpha}{2} A+\sum_{n \geq 2} \beta_{n} \frac{i^{n+1}}{n !} \frac{\partial^{n}}{\partial T^{n}} A+i \gamma \cdot\left(1+f_{R}\right)\left(1+\frac{i}{\omega_{0}} \frac{\partial}{\partial T}\right)\left(A(z, T) \int_{-\infty}^{\infty} R(\tau)|A(z, T-\tau)|^{2} d \tau\right)
$$

Ce logiciel de simulation a notamment été utilisé dans plusieurs travaux de recherche publiés récemment [2-3-7]. 


\section{Paramètres de simulation}

La cavité dans notre cas est constituée de cinq éléments qui sont :

Le Milieu amplificateur (EDF); Une fibre standard (SMF); Une fibre compensatrice (SDF); Un absorbant saturable (SA) et un coupleur de sortie (OC). Le tableau suivant résume les principaux paramètres utilisés dans la simulation:

Tableau 1. Caractéristiques de la cavité

\begin{tabular}{|l|c|c|c|c|c|}
\hline \multicolumn{1}{|c|}{ Eléments } & Pertes & gain & MFD & Longueur (m) & Dispersion (ps $\backslash \mathrm{nm} \backslash \mathrm{km})$ \\
\hline Milieu amplificateur (EDF) & 0 & 1,32 & 6 & 2,2 & $-12,5$ \\
\hline Fibre standard (SMF) & 0 & 0 & 10 & 10,4 & 17 \\
\hline Fibre compensatrice (SDF) & 0 & 0 & 10 & Variable & -91 \\
\hline Miroir de sortie (OC) & Variable & 0 & 7 & 0,01 & 0 \\
\hline
\end{tabular}

Dans les simulations, nous avons pris en compte les effets non linéaires d'automodulation de phase, de modulation de phase croisée, de mélange à quatre ondes ainsi que la diffusion Raman. Les effets de biréfringence ne sont pas pris en compte; nous considérons alors que la propagation s'effectue sur un seul axe de polarisation de la fibre.

L'impulsion injectée est caractérisée par: Une gaussienne avec $\lambda_{0}=1550 \mathrm{~nm}, \mathrm{~T}_{0}=0.15 \mathrm{ps}$ et $\mathrm{E}=100 \mathrm{pJ}$.

\section{Etude de l'influence de la longueur de la fibre compensatrice}

Dans cette partie nous avons varié la longueur de la fibre Compensatrice SDF de manière à obtenir différentes valeurs de la dispersion moyenne dans la cavité.

Pour calculer la longueur qui correspond à une valeur donnée de la dispersion, nous avons utilisé la formule suivante [8]:

$$
L_{S D F}=\left\lfloor 149.3-12.6 * D_{m o y}\right\rfloor /\left\lfloor 91.71+12.6 * D_{\text {moy }}\right\rfloor
$$

Pour chaque valeur de la dispersion, nous avons tracé plusieurs courbes qui représentent les différentes caractéristiques de l'impulsion. Nous allons présenter dans ce qui suit:

- La variation de l'énergie maximale en fonction de LSDF pour 1 tour de cavité.

- Evolution de la largeur spectrale. 
- La variation de $\lambda_{\text {Max }}$ pour 1 tour de cavité.

- La variation de $\mathrm{R}_{\lambda}=\lambda_{\text {Max }} / \lambda_{\text {Min }}$ pour 1 tour de cavité.

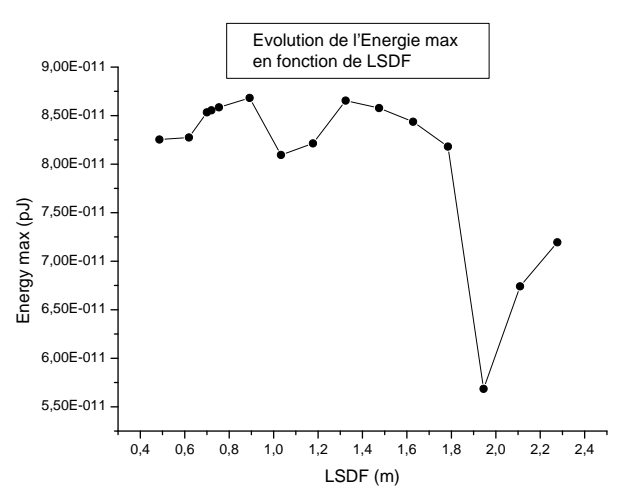

Fig.3. Variation de l'énergie maximale en fonction $\mathrm{L}_{\mathrm{SDF}}$

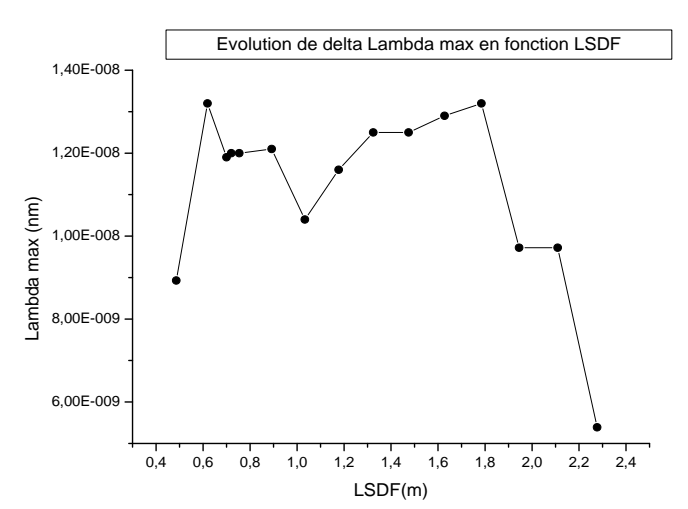

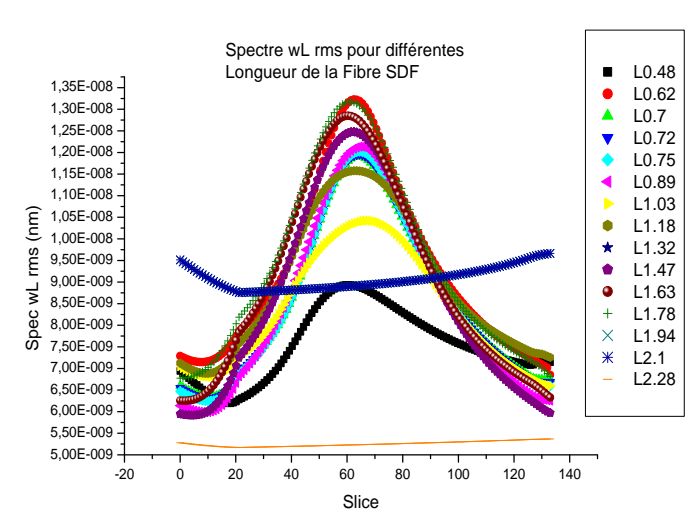

Fig.4. Evolution de la largeur spectrale pour les différentes valeurs de $\mathrm{L}_{\mathrm{SDF}}$

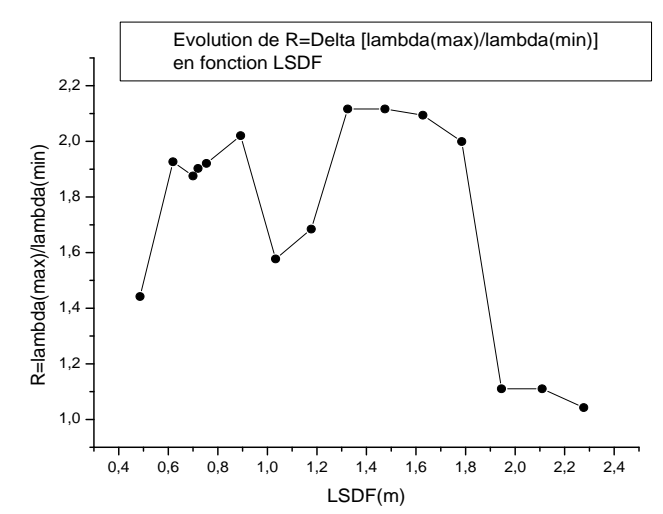

Fig.5. Variation de $\lambda_{\operatorname{Max}}$ et $\mathrm{R}_{\lambda}$ en fonction de $\mathrm{L}_{\mathrm{SDF}}$

Nous constatons que les meilleures performances sont obtenues pour une longueur de la fibre compensatrice variant entre 1.4 et $1.7 \mathrm{~m}$.

\section{Etude de l'influence des pertes}

Comme nous l'avons si bien constaté dans la $1^{\text {ère }}$ étude, nous avons fixé dans cette partie la longueur de la cavité à $\mathrm{L}_{\mathrm{SDF}}=1.63 \mathrm{~m}$ qui correspond à une dispersion nulle et nous avons varié les pertes dans l'intervalle [150,210]. Nous présentons dans ce qui suit:

- La variation de l'énergie maximale pour 1 tour de cavité.

- Evolution de la largeur spectrale

- La variation de $\lambda_{\text {Max }}$ pour 1 tour de cavité. 
- La variation de $\mathrm{R}_{\lambda}=\lambda_{\text {Max }} / \lambda_{\text {Min }}$ pour 1 tour de cavité.

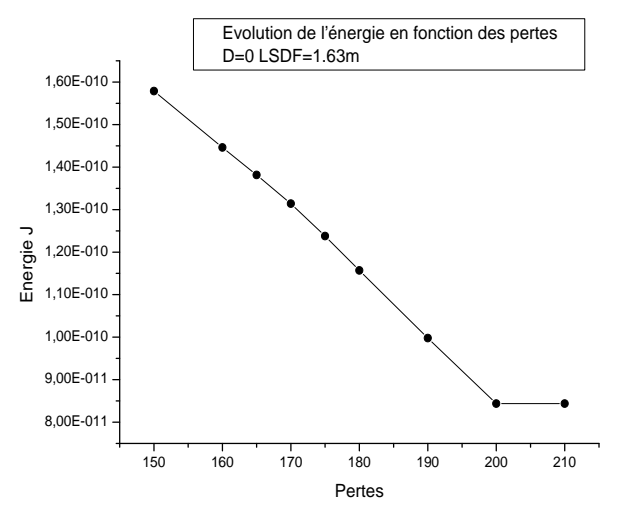

Fig.6. Variation de l'énergie maximale en fonction des pertes

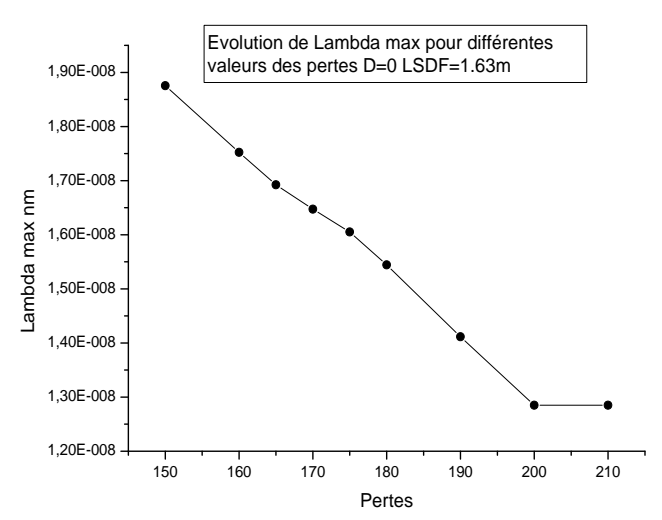

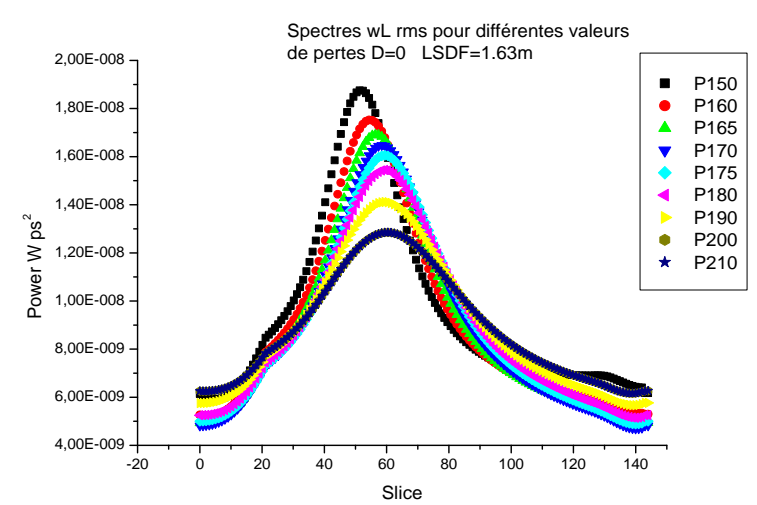

Fig.7. Evolution de la largeur spectrale pour les différentes valeurs des pertes

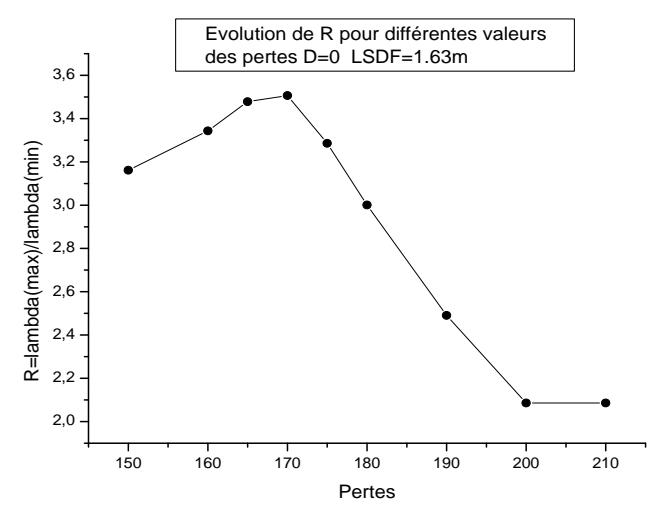

Fig.8. Variation de $\lambda_{\operatorname{Max}}$ et $\mathrm{R}_{\lambda}$ fonction des pertes

En changeant les paramètres de simulation, nous avons constaté qu'il existe un ensemble de paramètres pour lesquels le rapport $\mathrm{R}_{\lambda}=\lambda_{\text {Max }} / \lambda_{\text {Min }}$ est optimal. Le comportement de l'impulsion ressemble à une respiration tout au long du passage des différents éléments qui constituent la cavité. Le fait de trouver un optimum pour le facteur $\mathrm{R}_{\lambda}$ nous a poussés à varier d'autres paramètres afin de l'optimiser. Nous rappelons qu'optimiser le facteur $\mathrm{R}_{\lambda}$ revient à élargir la bande passante de la fibre.

\section{Etude de l'influence du taux de modulation de l'absorbant saturable}

Nous avons constaté que la courbe représentant le rapport $\mathrm{R}_{\lambda}$ passe par un optimum qui correspond à la valeur des pertes $\mathrm{P}=170$. Nous nous sommes placés en ce point et nous avons varié le taux de modulation de l'absorbant saturable R. Sachant que [8]: 


$$
R=R_{0}+R_{\text {sat }}-\frac{R_{\text {sat }}}{1+\frac{P}{P_{\text {sat }}}}
$$

Nous avons tracé les différentes caractéristiques en fonction de $\mathrm{R}_{0}$. Avec:

$\mathrm{R}_{\text {sat }}+\mathrm{R}_{0}=100 \%$.

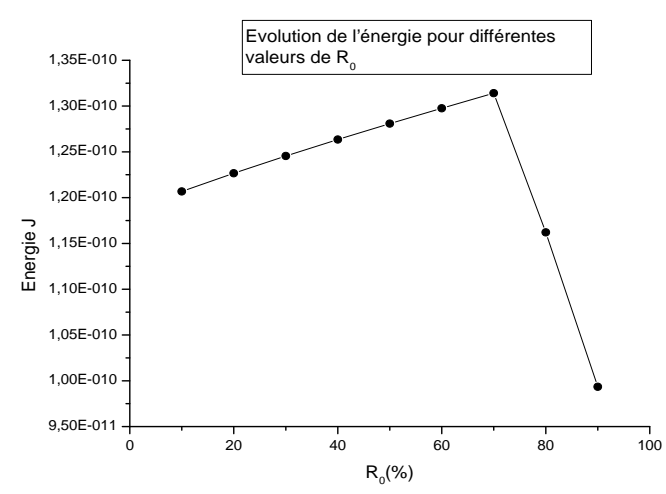

Fig.9. Variation de l'énergie maximale en fonction de $\mathrm{R}_{0}$

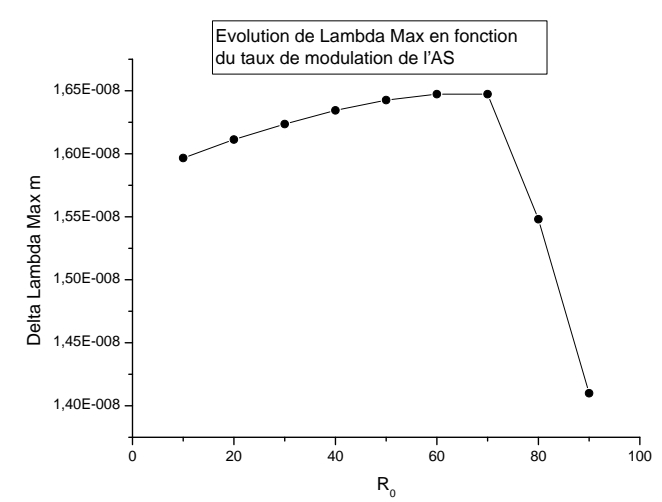

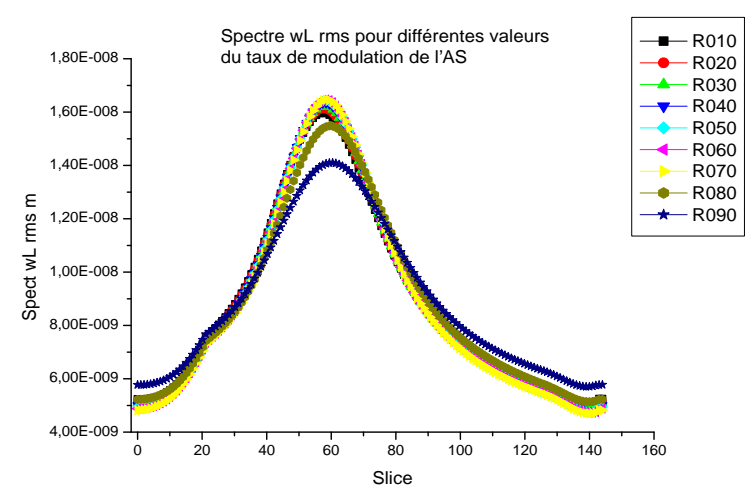

Fig.10. Evolution de la largeur spectrale en fonction de $\mathrm{R}_{0}$

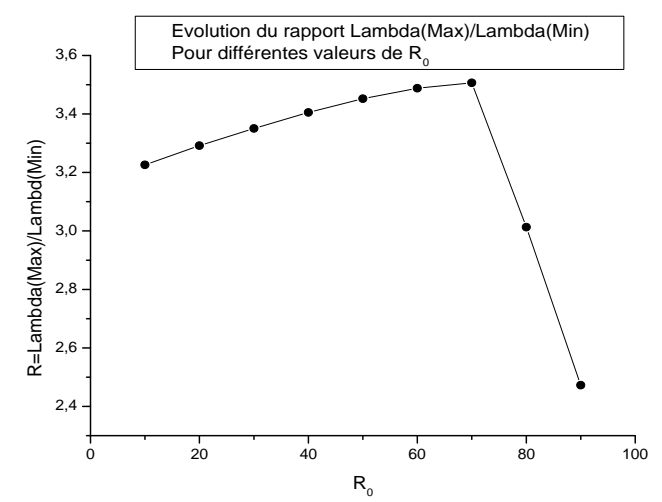

Fig.11. Variation de $\lambda_{\operatorname{Max}}$ et $\mathrm{R}_{\lambda}=\lambda_{\text {Max }} / \quad \lambda_{\text {Min }}$ en fonction $\mathrm{R}_{0}$

Nous remarquons que pour garder la valeur optimale de $\mathrm{R}_{\lambda}=3.4$, il faut maintenir le facteur de saturation de l'absorbant à une valeur précise qui est $\mathrm{R}_{0}=70$.

\section{CONCLUSION}

Nous avons montré à travers cette étude qu'il est possible de simuler la dynamique complexe de la propagation d'une impulsion courte dans une cavité fibrée en utilisant un logiciel se simulation et d'en tirer plusieurs informations. 
Malgré le fait qu'il n'est pas possible de toucher au programme de calcul, ce logiciel offre une variété de possibilité qui permet d'imaginer divers types de cavités et simuler par la suite le comportement d'une impulsion dans les différents éléments qui la constituent. Nous avons montré qu'un choix adéquat des paramètres permet d'élargir la bande passante en optimisant le rapport $\mathrm{R}_{\lambda}$.

\section{REFERENCES}

[1] Tombelaine V. (2007), Etude de rayonnements à large bande spectrale induits dans les fibres optiques microstructurées air-silice“ thèse de doctorat. Université de Limoges. [2] Schreiber et al. Supercontinuum generation by femtosecond single and dual wavelength pumping in photonic crystal fibers with two zero dispersion wavelengths. Opt. Expr. 2005, 13, 9556.

[3] Andersen et al. High repetition rate tunable femtosecond pulses and broadband amplification from fiber laser pumped parametric amplifier. Opt. Expr. 2006, 14, 4765. [4] Limpert et al. High-power femtosecond Yb-doped fiber amplifier. Opt. Expr. 2002, 10, 628 .

[5] Limpert et al. Ultra-broad bandwidth parametric amplification at degeneracy. Opt. Expr. 2005, 13, 7386.

[6] Schreiber et al. High average power supercontinuum generation in photonic crystal fibers. Opt. Comm. 2003, 228, 71.

[7] Schreiber et al. Microjoule-level all-polarization-maintaining femtosecond fiber source. Opt. Lett. 2006, 31, 574.

[8] Schreiber T., Ortaç B., Limpert J., Tünnermann A. On the study of pulse evolution in ultra-short pulse mode-locked fiber lasers by numerical simulations. Optics Express. June 2007, 15(13), 8262/25. 


\section{ÉTUDE DE LA PROPAGATION D'UNE IMPULSION COURTE EN CAVITE \\ LASER AVEC UN LOGICIEL DE SIMULATION NUMERIQUE}

\section{RESUME}

Le but de cette représentation est de montrer les potentialités (Rapidité de calcul, accès à la dynamique, faisabilité d'études systématique) de l'étude numérique de la dynamique non linéaire en cavité laser assistée par logiciel. Nous allons donner comme exemple, un type de cavité complètement fibrée constituée de plusieurs éléments et étudier par la suite les paramètres physiques d'une impulsion se propageant dans cette cavité, en déterminant ses caractéristiques à la sortie. Les résultats obtenus sont intéressants tout en projetant de les vérifier expérimentalement en réalisant des montages semblables à ce type de cavités.

Mots clés: Fibre optique, Impulsion courte, bande passante, largeur spectrale.

\section{How to cite this article}

Terniche S, Kellou A, Kermaoui A, Si Fodil R and Becheker R. Study of the propagation of short pulse laser with cavity using numerical simulation software. $\mathrm{J}$ Fundam Appl Sci. 2012, 4(1), 73-80. 